ISSN 0103-9954

\title{
NÍVEIS CRESCENTES DE SATURAÇÃO POR BASES E DESENVOLVIMENTO INICIAL DE TECA EM UM LATOSSOLO DE TEXTURA MÉDIA
}

\author{
INCREASING LEVELS OF BASE SATURATION AND INITIAL DEVELOPMENT OF \\ TEAK IN AN OXISOL OF MEDIUM TEXTURE
}

\author{
Lilian Guimarães de Favare ${ }^{1}$ Iraê Amaral Guerrini ${ }^{2}$ Clarice Backes ${ }^{3}$
}

\begin{abstract}
RESUMO
A teca é uma espécie florestal de alto valor comercial, porém, o pouco conhecimento do comportamento desta espécie em relação à acidez do solo é um fator importante que pode limitar o seu cultivo. O objetivo deste trabalho foi avaliar o efeito da elevação da saturação por bases do solo sobre o desenvolvimento inicial da teca (Tectona grandis L.f.). O experimento foi conduzido em estufa, em recipientes plásticos com capacidade de 50L. O delineamento experimental utilizado foi inteiramente casualizado, com cinco tratamentos e quatro repetições. Os tratamentos constituíram-se de: saturação por bases naturais do solo e os demais tratamentos com 20,40,60 e 80 \% de saturação. A correção do solo favoreceu todas as características de crescimento avaliadas nas plantas de teca. A calagem afetou positivamente a absorção de $\mathrm{N}, \mathrm{P}, \mathrm{K}, \mathrm{Ca}$, $\mathrm{Mg}, \mathrm{B}, \mathrm{Cu}$ e $\mathrm{Fe}$. A exigência nutricional da espécie obedeceu a seguinte ordem decrescente: $\mathrm{N}>\mathrm{Ca}>\mathrm{K}$ $>\mathrm{Mg}>\mathrm{P}>\mathrm{S}>\mathrm{Fe}>\mathrm{Mn}>\mathrm{B}>\mathrm{Zn}>\mathrm{Cu}$. Saturações por bases estimadas de $70 \%$ proporcionaram maior desenvolvimento das plantas.
\end{abstract}

Palavras-chave: Tectona grandis; calagem; crescimento; nutrição mineral.

\begin{abstract}
Teak is a forest species of high commercial value, but the little knowledge on the behavior of this species in relation to soil acidity is an important factor that may limit its cultivation. The aim of this study was to evaluate the effect of high base saturation on the soil on the initial development of teak (Tectona grandis L.f.). The experiment was conducted in a greenhouse, in plastic containers with capacity of 50 liters. The experimental design was randomized, with five treatments and four replications. The treatments consisted of: natural saturation of the soil and the other treatments with $20 \%, 40 \%, 60 \%$ and $80 \%$ of saturation. Correcting the soil favored the growth characteristics of all evaluated plant teak. The liming positively affected the uptake of $\mathrm{N}, \mathrm{P}, \mathrm{K}, \mathrm{Ca}, \mathrm{Mg}, \mathrm{B}, \mathrm{Cu}$ and $\mathrm{Fe}$, and the nutritional requirements of the species had the following order: $\mathrm{N}>\mathrm{Ca}>\mathrm{K}>\mathrm{Mg}>\mathrm{P}>\mathrm{S}>\mathrm{Fe}>\mathrm{Mn}>\mathrm{B}>\mathrm{Zn}>\mathrm{Cu}$. Base saturations above $70 \%$ showed higher plant development.
\end{abstract}

Keywords: Tectona grandis; liming; growth; mineral nutrition.

1. Engenheira Agrônoma, Doutoranda do Programa Ciência Florestal, Universidade Estadual Paulista "Julio de Mesquita Filho", Faculdade de Ciências Agronômicas, Campus de Botucatu, Rua José Barbosa de Barros, 1780, CEP 18610-307, Botucatu (SP). Bolsista CNPq. lilianfavare@hotmail.com

2. Engenheiro Florestal, Dr., Prof. Titular do Departamento de Recursos Naturais e Ciência do Solo, Universidade Estadual Paulista "Julio de Mesquita Filho"/UNESP, Faculdade de Ciências Agronômicas, Campus de Botucatu, Rua José Barbosa de Barros, 1780, CEP 18610-307, Botucatu (SP). Bolsista do CNPq. iguerrini@fca.unesp.br

3. Engenheira Agrônoma, Dra., Profa. da Universidade Estadual de Goiás/UnU de São Luís de Montes Belos, Rua da Saudade, 56, CEP 76000-000, São Luís de Montes Belos (GO). clarice.backes@ueg.br

Recebido para publicação em 10/05/2010 e aceito em 22/08/2011 


\section{INTRODUÇÃO}

Os plantios de teca (Tectona grandis L.f.) iniciaram-se nos países da Ásia Tropical, principalmente na Índia, Myanmar e Tailândia, cujo objetivo era compensar o esgotamento das populações naturais de teca que eram exploradas de forma extrativista (FIGUEIREDO et al., 2005). No Brasil, os primeiros plantios de teca datam do final da década de 60, introduzidos pela empresa Cáceres Florestal S.A., no município de Cáceres MT, onde as condições climáticas são semelhantes aos países de origem da espécie (RIOS, 2007). A precipitação média anual dos países de origem encontra-se na faixa de 1205 - $3750 \mathrm{~mm}$, com três meses de estação seca e temperatura média anual de 15 - $41{ }^{\circ} \mathrm{C}$ (OMBINA, 2008).

Devido à qualidade da madeira produzida, a teca apresenta grande valor comercial, podendo ser uma opção economicamente viável para as regiões que atendam às condições edafoclimáticas da espécie. Neste sentido, torna-se necessário o conhecimento sobre suas exigências nutricionais para uma adequada avaliação do seu potencial produtivo. Segundo Silva et al. (2007a), quando se pretende intensificar o cultivo de determinadas espécies é fundamental reduzir as limitações químicas, a fim de promover o crescimento com maior rapidez e aumentar o índice de estabelecimento das mudas no campo. Em estudo comparativo entre ambiente de cerrado e cultivo florestal com teca (Tectona grandis L.f.), Campolim (2006) observou que o manejo e uso dos solos em áreas florestais influenciam nos sítios de troca catiônica e nas propriedades químicas do solo, com a queda da acidez potencial e elevação da saturação de bases.

Em virtude das baixas concentrações e reservas de Ca na maioria dos solos usados para fins de reflorestamentos, as fertilizações devem prever a aplicação de consideráveis quantidades de $\mathrm{Ca}$, junto com fontes de $\mathrm{N}, \mathrm{P}$ e $\mathrm{K}$ ou por meio de calagem (BEHLING, 2009). Isso se deve aos elevados teores de alumínio e, em alguns casos, de manganês (RAIJ, 1991). Portanto, o uso de corretivos e fertilizantes é uma prática fundamental no desenvolvimento das espécies florestais, não só para a redução da acidez do solo, mas também como fonte de nutrientes indispensáveis ao crescimento inicial das plantas.

Segundo Vale et al. (1996), é importante ressaltar que a resposta das plantas à prática da calagem pode ou não ocorrer, dependendo das características de cada uma das espécies que se pretende produzir, principalmente no que se refere à tolerância à acidez. As respostas das plantas, em relação aos efeitos da acidez no solo, não permitem generalizações e dificultam o estabelecimento de faixas de $\mathrm{pH}$ adequadas para as diversas culturas (RAIJ, 1991).

$\mathrm{O}$ efeito da calagem no desenvolvimento de teca pode estar diretamente relacionado ao fornecimento de $\mathrm{Ca}$, pois segundo Takle e Mujundar (1957), citados por Matricardi (1989), diversos estudos identificam a teca como uma espécie calcícola, mostrando que ela necessita de uma quantidade relativamente grande deste nutriente para o seu desenvolvimento. Silva et al. (2000) também verificaram a exigência da cultura por $\mathrm{Ca}$ ao avaliar o estado nutricional de dois plantios de teca, com 9 e 17 anos, onde verificaram maior crescimento em altura em solos com maiores teores deste nutriente. $\mathrm{Na}$ ausência de cálcio, Barroso et al. (2005) observaram redução drástica do crescimento, clorose internerval, encarquilhamento e necrose das folhas, morte da gema apical, paralisação de emissão de raízes novas, apodrecimento das raízes secundárias, com consequente redução na produção de biomassa seca de plantas.

A influência da calagem tem sido estudada para diversas espécies florestais e com resultados satisfatórios para espécies como ipê-roxo (CRUZ et al., 2004), sumaúma (TUCCI et al., 2004), mogno (BARROS, 2001; PINTO et al., 2000, SILVA et al., 2007a), eucalipto (SILVA et al., 2007b), porém, para a teca não foram relatados no Brasil trabalhos demonstrando o efeito da elevação da saturação por bases no desenvolvimento e crescimento das plantas.

Visto o potencial da espécie e a escassa existência de pesquisas sobre o assunto, o objetivo deste trabalho foi avaliar o efeito da elevação da saturação por bases no desenvolvimento inicial de teca (Tectona grandis L.f.).

\section{MATERIAL E MÉTODOS}

O experimento foi conduzido em estufa plástica, sem controle de temperatura e umidade relativa do ar, na área experimental do Departamento de Recursos Naturais/Ciência do Solo, da Faculdade de Ciências Agronômicas de Botucatu-SP. O município está localizado na região Centro-Sul do Estado de São Paulo, nas coordenadas 22052'20"S e $48^{\circ} 26^{\prime} 37^{\prime \prime} \mathrm{W}$ e altitude de $804 \mathrm{~m}$.

O tipo de solo utilizado é classificado 
como Latossolo Vermelho-Escuro, textura média, conforme Embrapa (2006), coletado em área desbastada de eucalipto, na FCA/UNESP, na profundidade de 0,20 a $0,40 \mathrm{~m}$. De acordo com o resultado da análise, o solo possui as seguintes características químicas: $\mathrm{pH}\left(\mathrm{CaCl}_{2}\right)$ de 4,1 ; $19 \mathrm{~g} \mathrm{dm}^{-3}$ de M.O.; $2 \mathrm{mg} \mathrm{dm}^{-3}$ de P (resina) e 15; 64; 0,$3 ; 1 ; 0$ e $66 \mathrm{mmol}_{\mathrm{c}} \mathrm{dm}^{-3}$ de, respectivamente, $\mathrm{Al}^{+3}$, $\mathrm{H}^{+}+\mathrm{Al}^{+3}, \mathrm{~K}, \mathrm{Ca}, \mathrm{Mg}$, SB e CTC, além de 0,$26 ; 1,0$; 59; 0,3 e $0,1 \mathrm{mg} \mathrm{dm}^{-3}$ de, respectivamente, $\mathrm{B}, \mathrm{Cu}$, $\mathrm{Fe}, \mathrm{Mn}$ e Zn, e saturação por bases $(\mathrm{V} \%)$ de $2 \%$. As características físicas são: $66 \%$ de areia, $7 \%$ de silte e $27 \%$ de argila.

A espécie utilizada foi a teca (Tectona grandis L.f.), clone fornecido pela empresa Floresteca S/A situada no município de CuiabáMT. O delineamento experimental utilizado foi o inteiramente casualizado, com seis tratamentos e quatro repetições. Os tratamentos constituíramse de: saturação por bases naturais do solo e os demais tratamentos com 20, 40, 60 e $80 \%$ de saturação, correspondendo às doses de $0,29,66$, 103, $139 \mathrm{~g} \mathrm{vaso}^{-1}$ de calcário dolomítico com PRNT de $92 \%$. Após a aplicação do corretivo, o solo ficou incubado por 30 dias, mantendo-se a umidade gravimétrica do solo próxima à capacidade de campo $(12 \%)$. A umidade do solo foi mantida repondo a água perdida $(500 \mathrm{ml})$, inicialmente a cada 2 dias e, posteriormente, diariamente, em função do aumento de evapotranspiração, inclusive para a testemunha que não recebeu calcário.

As mudas com 120 dias de idade foram plantadas em novembro de 2008, em recipientes plásticos com capacidade de $50 \mathrm{~L}$, preenchidos com o solo respectivo de cada tratamento. A irrigação, realizada com regador, foi realizada diariamente de forma a manter a umidade do solo a $80 \%$ de saturação. Todos os tratamentos receberam $80 \mathrm{mg}$ $\mathrm{dm}^{-3} \mathrm{de} \mathrm{N}, 150 \mathrm{mg} \mathrm{dm}^{-3}$ de $\mathrm{P}_{2} \mathrm{O}_{5}, 80 \mathrm{mg} \mathrm{dm}^{-3} \mathrm{de} \mathrm{K}_{2} \mathrm{O}$, $5,29 \mathrm{mg} \mathrm{dm}^{-3}$ de B e 4,76 $\mathrm{mg} \mathrm{dm}^{-3} \mathrm{de} \mathrm{Zn}$, utilizando como fontes, respectivamente, ureia, superfosfato simples, cloreto de potássio, ácido bórico e sulfato de zinco.

As características de crescimento foram avaliadas mensalmente por meio de medidas de altura de plantas, desde o colo até o ápice das plantas com o auxílio de régua graduada, e do diâmetro do caule, utilizando-se de um paquímetro digital.

Aos 240 dias após o plantio realizou-se a avaliação do Índice Relativo de Clorofila (IRC) utilizando-se o medidor portátil Chlorophyll Meter, modelo SPAD-502, e as taxas fotossintéticas (TF) e taxas de transpiração (TT) com um analisador automático portátil com sistema fechado Li-Cor LI-6400XT, IRGA. Estas determinações foram realizadas durante o período da manhã, entre nove e dez horas, no par de folhas situadas na região central da planta, e com três leituras por folha.

Ao final de oito meses todas as plantas foram separadas em folhas, caule e raízes, lavadas e colocadas em estufa de circulação forçada de ar por 72 horas, na temperatura de $65^{\circ} \mathrm{C}$, até a obtenção de peso constante. A partir do peso seco das partes da planta, foi calculada a biomassa total.

Após a secagem e pesagem, as folhas e caules foram moídas e enviadas para o Laboratório de Nutrição de Plantas da Faculdade de Ciências Agronômicas (FCA) da UNESP para a determinação da concentração de macro e micronutrientes, de acordo com Malavolta et al. (1997). O acúmulo de nutrientes nas plantas foi calculado pela multiplicação da biomassa seca de cada órgão pelos respectivos teores de nutrientes.

Os resultados obtidos foram submetidos à análise de variância e à posterior análise de regressão, ajustando-se as equações aos dados obtidos em função das saturações por base. Foi adotada como critério na escolha do modelo a interação pelo teste $\mathrm{F}$ significativo a $5 \%$ e $1 \%$, com auxílio do programa SISVAR 4.2.

\section{RESULTADOS E DISCUSSÃO}

Para a altura de plantas, houve influência dos tratamentos somente a partir dos 150 dias após o plantio (dap). Nesta época, verificou-se que a altura de plantas ajustou-se de forma linear crescente às diferentes saturações por bases (Figura 1). Nas demais épocas, mesmo com o efeito quadrático, a maior altura de plantas foi obtida com o maior V\% (80\%).

Para o diâmetro do caule, também foi observada a influência dos tratamentos após 150 dap, apresentando efeito quadrático em função dos tratamentos aos 150 e 180 dap. Nessas épocas, os valores estimados de V\% (50 e $54 \%$ ) resultaram nos maiores diâmetros de caule. Nas demais épocas (210 e 240 dap) houve efeito linear crescente em função dos níveis crescentes de V\% (Figura 2), indicando valores ideais de V\% acima de $80 \%$. Campolin (2006), em experimento comparando as características físicas e químicas do solo influenciadas pelos plantios de teca em comparação com cerrado nativo, usou saturação por bases de 
a)

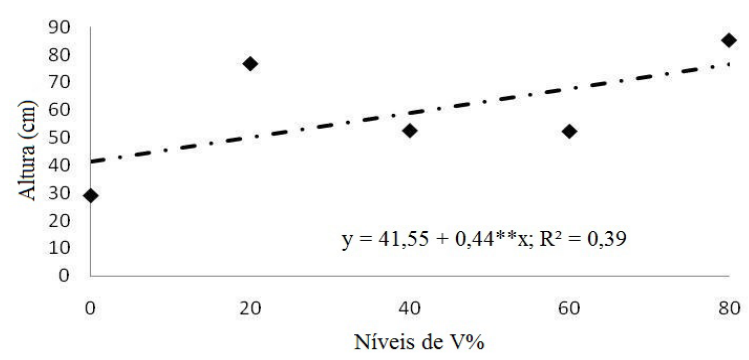

c)

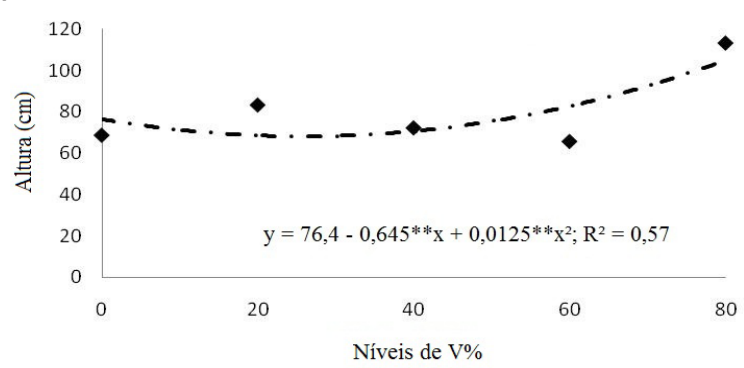

b)

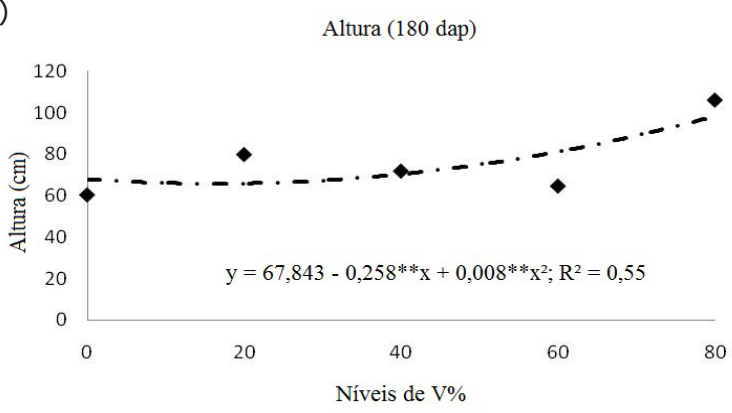

d)

Altura (240 dap)

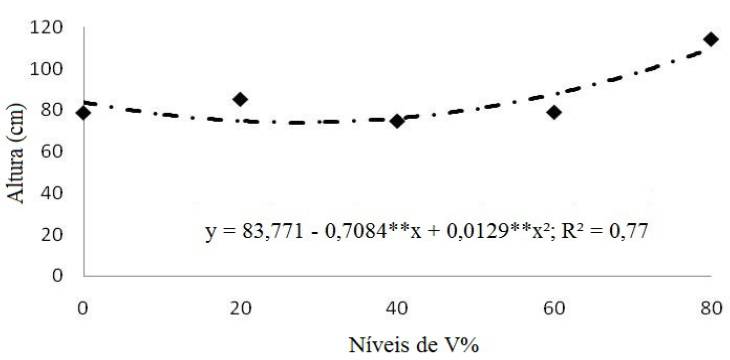

FIGURA 1: Altura das plantas de teca 150 (a), 180 (b), 210 (c), 240 (d) dias após plantio (dap), em função de níveis crescentes de saturação por bases (V\%).

FIGURE 1: Height of teak plants 150 (a), 180 (b), 210 (c), 240 (d) days after planting (dap), due to increasing levels of base saturation $(\mathrm{V} \%)$.
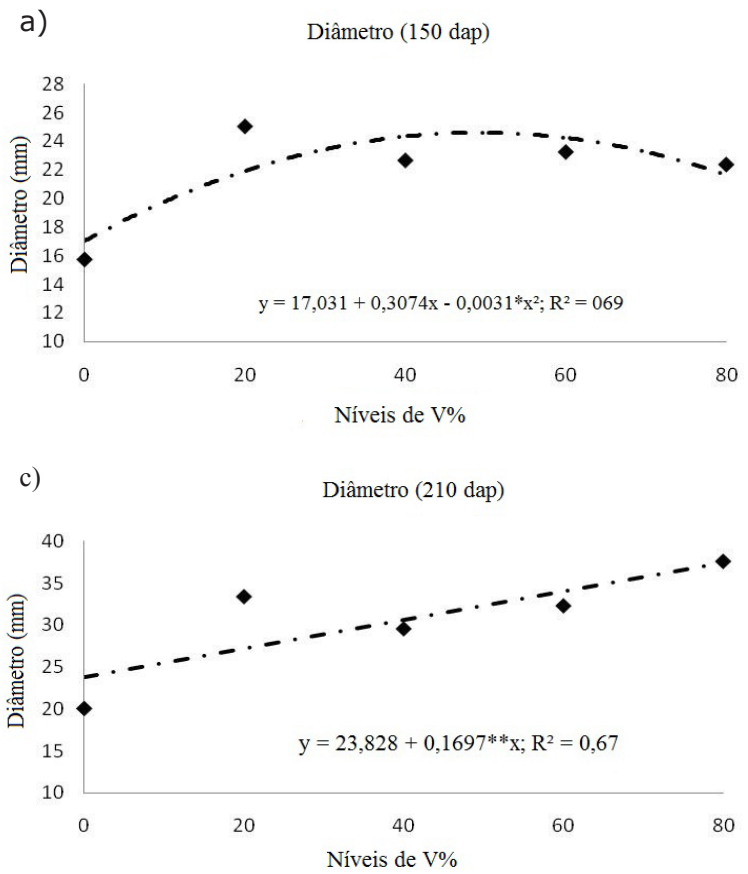

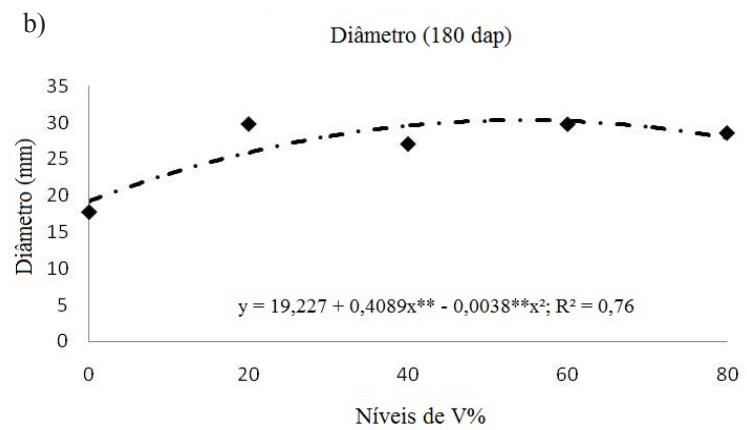

d) Diâmetro (240 dap)

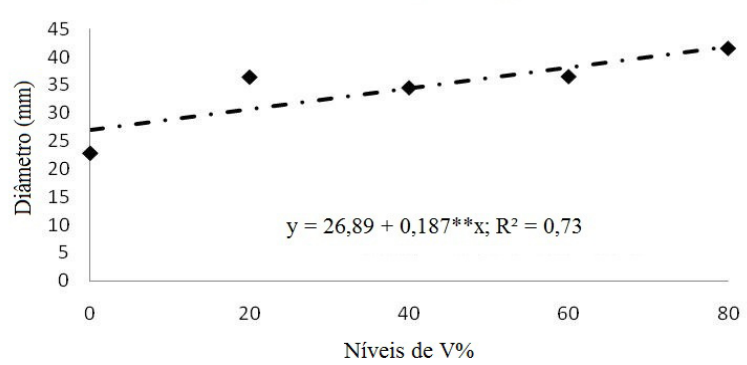

FIGURA 2: Diâmetro do caule de plantas de teca 150 (a), 180 (b), 210 (c), 240 (d) dias após plantio (dap), em função de níveis crescentes de saturação por bases $(\mathrm{V} \%)$.

FIGURE 2: Stem diameter of teak plants 150 (a), 180 (b), 210 (c), 240 (d) days after planting (dap), due to increasing levels of base saturation $(\mathrm{V} \%)$. 
$65 \%$ como recomendação para teca. Respostas positivas da calagem sobre a altura e o aumento do diâmetro do caule também foram observadas para mudas de Tabebuia impetiginosa - ipê-roxo (CRUZ et al., 2004) e Ceiba pentandra - sumaúma (TUCCI et al., 2004). Barros (2001) e Pinto et al. (2000) observaram respostas positivas com a aplicação da calagem sobre a altura das plantas de mogno.

Os diferentes níveis de saturação por bases influenciaram significativamente na biomassa seca das partes e do total da planta de teca (Figura 3). De acordo com as equações ajustadas, o maior acúmulo de biomassa seca das folhas, raízes e total, foi obtido, respectivamente, com V\% de 67, 54 e $60 \%$. Para a biomassa seca de caule, embora tenha sido ajustado o modelo quadrático, a saturação que proporcionou o máximo acúmulo está acima das estudadas. Dessa forma, o maior acúmulo de biomassa seca de caule foi obtido com a saturação acima de $80 \%$. O tratamento de maior saturação por bases $(80 \%)$ aos 240 dap apresentou a seguinte proporção de produção de biomassa seca: $26 \%$ de folhas, $25 \%$ de caule e $49 \%$ de raiz.

A resposta à calagem observada pelo aumento da biomassa seca revela os efeitos negativos da acidez no desenvolvimento das plantas

a)

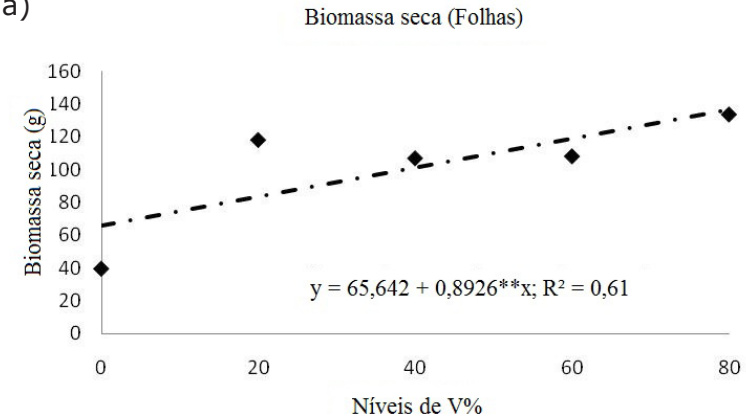

c)

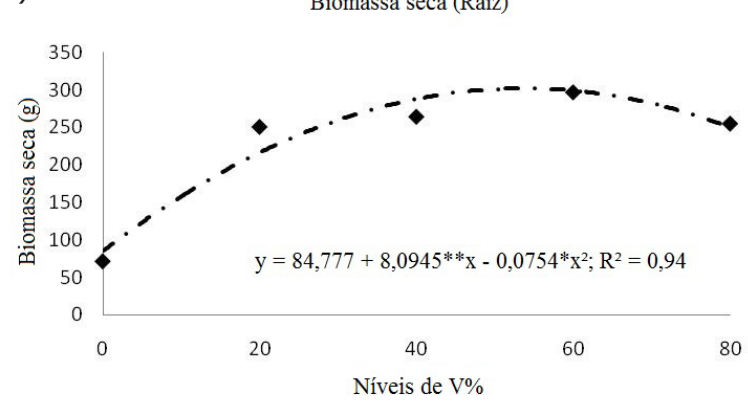

de teca. As possíveis variações do $\mathrm{pH}$ do solo, em função das diferentes saturações por bases, podem ter proporcionado influência indireta no desenvolvimento das plantas por interferirem na disponibilidade de nutrientes no solo. Além disso, a prática da calagem exerce papel importante no fornecimento de cálcio e magnésio como nutrientes. Segundo Vale et al. (1996), a deficiência de cálcio limita o crescimento do sistema radicular, podendo ser mais restritiva que a toxidez de alumínio. Como o solo utilizado apresentou baixo teor inicial de $\mathrm{Ca}$ $\left(1 \mathrm{mmol}_{\mathrm{c}} \mathrm{dm}^{-3}\right)$, a adição de calcário justifica os resultados positivos no acúmulo de biomassa seca de raízes e, consequentemente, da biomassa seca da parte aérea.

Em vários trabalhos foram encontrados resultados positivos quando do uso de corretivo pela elevação da concentração de $\mathrm{Ca}^{2+} \mathrm{e} \mathrm{Mg}^{2+}$ trocáveis e diminuição da acidez do solo (SILVA et al., 2007a; SILVA et al., 2008; TUCCI et al., 2007), o que favorece o desenvolvimento do sistema radicular. Barroso et al. (2005), trabalhando com solução nutritiva, também verificaram efeito positivo do $\mathrm{Ca}$ em plantas de teça, com diminuição da biomassa seca da parte aérea pela omissão de Ca, resultando em redução de 80 \% em relação à solução completa.
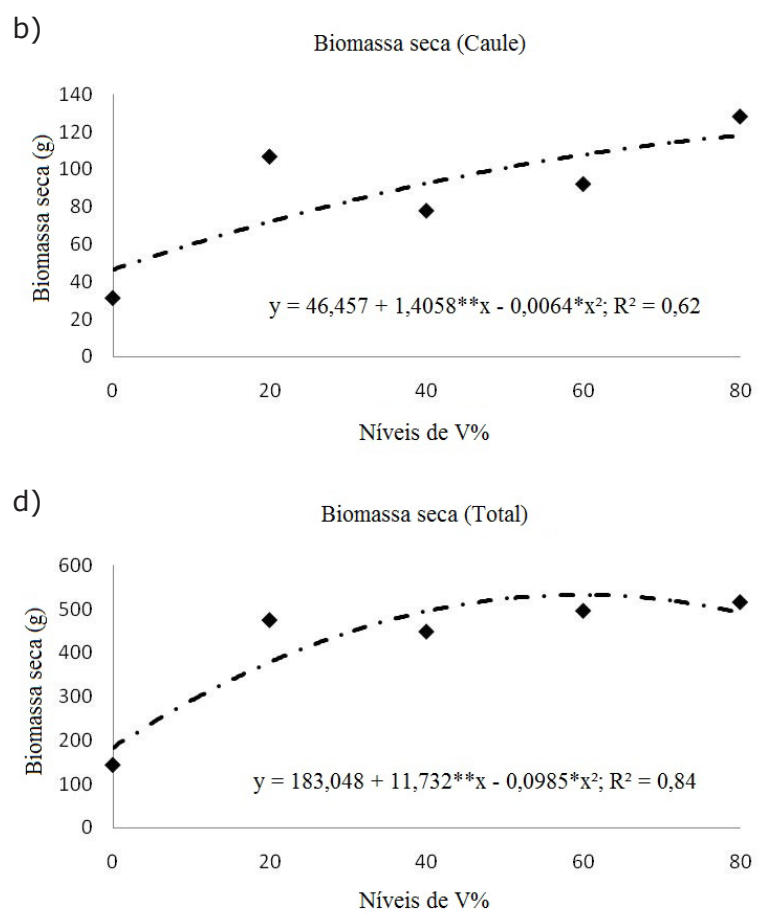

FIGURA 3: Biomassa seca das folhas (a), do caule (b), da raiz (c) e total (d) de plantas de teca com 8 meses de idade, em função de níveis crescentes de saturação por bases (V\%).

FIGURE 3: Dry weight of leaves (a), stem (b), roots (c) and total (d) of teak tree with 8 months of age, due to increasing levels of base saturation $(\mathrm{V} \%)$. 
Furtini Neto et al. (1999) também verificaram aumento na biomassa seca total de várias espécies em função da calagem. Segundo esses autores, estes resultados são importantes na medida em que se ressaltam diferentes reações das espécies florestais às condições de acidez do solo e à capacidade de resposta dessas espécies à aplicação de corretivos. Segundo Campolin (2006), o aumento dos valores de $\mathrm{pH}$ do solo em área sob plantio de teca contribuiu para a manutenção dos cátions trocáveis como o cálcio, magnésio e o potássio do solo.

Com relação à nutrição mineral das plantas, verificou-se, quanto aos macronutrientes, influência dos tratamentos para $\mathrm{K}, \mathrm{Ca}$ e $\mathrm{Mg}$ (Tabela 1). Para o K verificou-se redução da concentração com o aumento da saturação por bases, devido ao efeito de diluição, visto que a elevação do $\mathrm{V} \%$ proporcionou aumento da biomassa seca de folhas. As concentrações de $\mathrm{Ca}$ e $\mathrm{Mg}$ ajustaram-se de forma quadrática, e segundo as equações ajustadas (Ca: y $=3,82857+0,448393 \mathrm{x}+0,004152 \mathrm{x}^{2}$ e Mg: $\mathrm{y}=0,99$ $\left.+0,1142 x-0,000813 x^{2}\right)$, as saturações estimadas de 54 e $70 \%$ proporcionaram, respectivamente, os máximos valores de $\mathrm{Ca}$ e $\mathrm{Mg}$. Para a concentração de micronutrientes nas folhas, verificou-se influência apenas para $\mathrm{Mn}$ e Zn, com redução na concentração com o aumento da saturação por bases.

Mesmo com os efeitos favoráveis da calagem na decomposição da matéria orgânica, com consequente aumento na mineralização do $\mathrm{N}$ e na disponibilidade de $\mathrm{P}$, não foi observada a influência dos tratamentos na concentração destes nutrientes nas folhas de teca. Este fato pode ser atribuído aos baixos teores de matéria orgânica no solo utilizado e também à menor fixação de $\mathrm{P}$ em solos de textura mais arenosa, pois todos os tratamentos receberam as mesmas doses de fertilizantes nitrogenados e fosfatados.

Para a concentração de nutrientes no caule das plantas, houve influência dos tratamentos apenas para N, Ca, Mg, Mn e Zn (Tabela 2). Para $\mathrm{N}$ verificou-se efeito quadrático com diminuição e posterior aumento da concentração em função do aumento das saturações por bases. A máxima concentração de $\mathrm{Ca}$ foi obtida com a saturação estimada de $53 \%$, em função da equação ajustada $\left(\mathrm{y}=4,1714-0,4529 \mathrm{x}-0,0043 \mathrm{x}^{2}\right)$. Para os demais nutrientes, os efeitos foram lineares, crescente para o Mg e decrescente para Mn e Zn. Silva et al. (2007a), testando doses crescentes de corretivo na formação de mudas de mogno, verificaram que a calagem afetou positivamente a absorção de $\mathrm{N}, \mathrm{P}, \mathrm{K}, \mathrm{Ca}$, $\mathrm{Mg}, \mathrm{Mn}$ e negativamente de Fe. Furtunato (2001) observou que a calagem do solo proporcionou aumento na concentração de $\mathrm{N}, \mathrm{Ca}$ e $\mathrm{Mg}$ nas raízes e parte aérea de grápia (Apuleia leiocarpa), bem como $\mathrm{K}$ na parte aérea, e diminuiu a concentração de $\mathrm{Mn}$ nas raízes ve parte aérea.

Os níveis de saturação por bases não influenciaram de forma significativa as concentrações de $\mathrm{B}, \mathrm{Cu}$ e $\mathrm{Fe}$ nas folhas e no caule das plantas de teca. Esperava-se uma redução na absorção de $\mathrm{Cu}$ e Fe com o aumento das doses de corretivo, pois quanto maior o valor do $\mathrm{pH}$, maior é a possibilidade de precipitação desses micronutrientes. Esse fato sugere que o aumento do $\mathrm{pH}$ não foi suficiente

TABELA 1: Concentração de nutrientes nas folhas de teca 240 dap, em função de níveis crescentes de saturação por bases (V\%). Botucatu-SP (julho/2009).

TABLE 1: Nutrient concentration in leaves of teak 240 dap, due to increasing levels of base saturation (V\%). Botucatu-SP (July/2009).

\begin{tabular}{|c|c|c|c|c|c|c|c|c|c|c|c|}
\hline \multirow{3}{*}{$\begin{array}{l}\text { Níveis de } \\
\text { saturação } \\
\text { por bases }\end{array}$} & \multicolumn{11}{|c|}{ Concentração de nutrientes nas folhas } \\
\hline & \multicolumn{11}{|c|}{ Nutrientes } \\
\hline & $\mathrm{N}$ & $\mathrm{P}$ & K & $\mathrm{Ca}$ & $\mathrm{Mg}$ & $\mathrm{S}$ & $\mathrm{B}$ & $\mathrm{Cu}$ & $\mathrm{Fe}$ & $\mathrm{Mn}$ & $\mathrm{Zn}$ \\
\hline ------\%"----- & \multicolumn{6}{|c|}{ - } & \multicolumn{5}{|c|}{------------------mg kg } \\
\hline 0 & 20 & 1,4 & 15 & 4 & 0,88 & 1,2 & 78 & 7 & 637 & 139 & 18 \\
\hline 20 & 17 & 1,3 & 8 & 12 & 3,15 & 1,0 & 63 & 7 & 587 & 385 & 17 \\
\hline 40 & 17 & 1,2 & 7 & 15 & 4,35 & 1,1 & 75 & 8 & 468 & 160 & 10 \\
\hline 60 & 17 & 1,5 & 6 & 16 & 4,60 & 1,0 & 70 & 6 & 497 & 89 & 8 \\
\hline 80 & 18 & 1,6 & 8 & 13 & 5,08 & 1,1 & 55 & 6 & 585 & 52 & 10 \\
\hline Regressão & ns & $\mathrm{ns}$ & $\mathrm{L}^{* *}, \mathrm{Q}^{* *}$ & $\mathrm{~L}^{* *}, \mathrm{Q}^{* *}$ & $\mathrm{~L}^{* *}, \mathrm{Q}^{*}$ & $\mathrm{~ns}$ & $\mathrm{~ns}$ & $\mathrm{~ns}$ & ns & $\mathrm{L}^{* *}, \mathrm{Q}^{*}$ & $\mathrm{~L}^{* *}$ \\
\hline
\end{tabular}

Em que: $\mathrm{ns}=$ não significativo; $*$ significativo a $5 \% ; *$ significativo a $1 \% ; \mathrm{L}=$ linear; $\mathrm{Q}=$ quadrática. 
TABELA 2: Concentração de nutrientes no caule de plantas de teca 240 dap, em função de níveis crescentes de saturação por bases (V\%). Botucatu-SP (julho/2009).

TABLE 2: Concentration of nutrients in the stems of plants weave 240 dap, due to increasing levels of base saturation (V\%). Botucatu-SP (July/2009).

\begin{tabular}{|c|c|c|c|c|c|c|c|c|c|c|c|}
\hline \multirow{3}{*}{$\begin{array}{c}\text { Níveis de } \\
\text { saturação por } \\
\text { bases }\end{array}$} & \multicolumn{11}{|c|}{ Concentração de nutrientes no caule } \\
\hline & \multicolumn{11}{|c|}{ Nutrientes } \\
\hline & $\mathrm{N}$ & $\mathrm{P}$ & $\mathrm{K}$ & $\mathrm{Ca}$ & $\mathrm{Mg}$ & $\mathrm{S}$ & $\mathrm{B}$ & $\mathrm{Cu}$ & $\mathrm{Fe}$ & $\mathrm{Mn}$ & $\mathrm{Zn}$ \\
\hline -----\%"---- & \multicolumn{6}{|c|}{-------------------g kg } & \multicolumn{5}{|c|}{-------------------'mg kg-1--------------. } \\
\hline 0 & 8 & 0,65 & 7 & 10 & 1,98 & 0,95 & 58 & 9 & 355 & 74 & 34 \\
\hline 20 & 6 & 0,60 & 7 & 9 & 3,33 & 0,88 & 54 & 8 & 97 & 65 & 26 \\
\hline 40 & 6 & 0,65 & 7 & 12 & 3,58 & 0,95 & 58 & 8 & 79 & 28 & 17 \\
\hline 60 & 5 & 0,73 & 7 & 11 & 3,90 & 0,88 & 59 & 7 & 181 & 15 & 13 \\
\hline 80 & 7 & 0,80 & 7 & 9 & 4,33 & 0,93 & 49 & 7 & 129 & 13 & 13 \\
\hline Regressão & $\mathrm{Q}^{* *}$ & ns & $\mathrm{ns}$ & $\mathrm{Q}^{* *}$ & $\mathrm{~L}^{*}$ & $\mathrm{~ns}$ & ns & $\mathrm{ns}$ & $\mathrm{ns}$ & $\mathrm{L}^{* *}$ & $\mathrm{~L}^{* *}$ \\
\hline
\end{tabular}

Em que: $\mathrm{ns}=$ não significativo; $*$ = significativo a $5 \%$;* $=$ significativo a $1 \% ; \mathrm{L}=$ linear; $\mathrm{Q}=$ quadrática.

para reduzir a disponibilidade de $\mathrm{Cu}, \mathrm{Fe}$ e $\mathrm{Zn}$ para plantas de teca. Este efeito de redução da absorção foi verificado apenas para as concentrações de $\mathrm{Mn}$ e Zn.

Os níveis crescentes de saturação por bases também influenciaram significativamente as quantidades acumuladas dos nutrientes na parte aérea das plantas de teca (Tabela 3). Para N, P, K, Mg, S e Fe, verificou-se efeito linear crescente em função das saturações por bases. Os máximos acúmulos de nutrientes na parte aérea das plantas com a maior saturação por bases $(80 \%)$ foram de 3,$2 ; 0,31 ; 1,8$; 1,2 e 0,3 g planta $^{-1}$ de, respectivamente, $\mathrm{N}, \mathrm{P}, \mathrm{K}, \mathrm{M} \mathrm{e}$
$\mathrm{S}$, e $94 \mathrm{mg}^{\text {planta }}{ }^{-1}$ de Fe. Estes resultados se devem ao aumento da biomassa seca e da concentração de nutrientes nas folhas e caules das plantas de teca.

As quantidades acumuladas de $\mathrm{Ca}, \mathrm{B}$, $\mathrm{Cu}$ e $\mathrm{Mn}$ ajustaram-se de forma quadrática às saturações por bases. Verificou-se que, segundo as equações ajustadas $(\mathrm{Ca}: \mathrm{y}=0,657786+0,076359 \mathrm{x}$ - $0,000629 x^{2} ; B: y=5,954+0,28065 x-0,00245 x^{2}$; $\mathrm{Cu}: \mathrm{y}=0,753714+0,02957 \mathrm{x}-0,000243 \mathrm{x}^{2}$ e $\mathrm{Mn}$ : $\left.\mathrm{y}=8,4714+0,4374 \mathrm{x}-0,0057 \mathrm{x}^{2}\right)$, as saturações estimadas de $61,57,61$ e $38 \%$ proporcionaram os máximos acúmulos de, respectivamente, $\mathrm{Ca}, \mathrm{B}, \mathrm{Cu}$ e $\operatorname{Mn}\left(3,0\right.$ g planta $^{-1}$ e $14 ; 1,6$ e 17 mg planta $\left.^{-1}\right)$.

TABELA 3: Acúmulo de nutrientes na parte aérea das plantas de teca 240 dap, em função de níveis crescentes de saturação por bases (V\%). Botucatu-SP (julho/2009).

TABLE 3: Nutrient accumulation in the shoots of teak 240 dap, due to increasing levels of base saturation (V\%). Botucatu-SP (July/2009).

\begin{tabular}{|c|c|c|c|c|c|c|c|c|c|c|c|}
\hline \multirow{3}{*}{$\begin{array}{l}\text { Níveis de } \\
\text { saturação } \\
\text { por bases }\end{array}$} & \multicolumn{11}{|c|}{ Acúmulo de nutrientes na parte aérea } \\
\hline & \multicolumn{11}{|c|}{ Nutrientes } \\
\hline & $\mathrm{N}$ & $\mathrm{P}$ & $\mathrm{K}$ & $\mathrm{Ca}$ & $\mathrm{Mg}$ & $\mathrm{S}$ & $\mathrm{B}$ & $\mathrm{Cu}$ & $\mathrm{Fe}$ & $\mathrm{Mn}$ & $\mathrm{Zn}$ \\
\hline ------0\%------- & \multicolumn{6}{|c|}{ 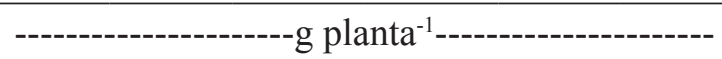 } & \multicolumn{5}{|c|}{--------------------'mg kg ${ }^{-1}$--------------.- } \\
\hline 0 & 1,0 & 0,08 & 0,8 & 0,5 & 0,1 & 0,1 & 4,9 & 0,6 & 39 & 7,9 & 1,8 \\
\hline 20 & 2,7 & 0,22 & 1,6 & 2,4 & 0,7 & 0,2 & 12,9 & 1,7 & 80 & 15,4 & 4,8 \\
\hline 40 & 2,3 & 0,18 & 1,3 & 2,5 & 0,8 & 0,2 & 12,4 & 1,5 & 57 & 19,0 & 2,4 \\
\hline 60 & 2,3 & 0,22 & 1,3 & 2,8 & 0,9 & 0,2 & 12,8 & 1,3 & 72 & 11,1 & 2,2 \\
\hline 80 & 3,2 & 0,31 & 1,8 & 2,9 & 1,2 & 0,3 & 13,5 & 1,7 & 94 & 8,5 & 2,9 \\
\hline Regressão & $\mathrm{L}^{* *}$ & $\mathrm{~L}^{* *}$ & $\mathrm{~L}^{* *}$ & $\mathrm{~L}^{* *}, \mathrm{Q}^{*}$ & $\mathrm{~L}^{* *}$ & $\mathrm{~L}^{* *}$ & $\mathrm{~L}^{* *}, \mathrm{Q}^{* *}$ & $\mathrm{~L}^{* *}, \mathrm{Q}^{*}$ & $\mathrm{~L}^{*}$ & $\mathrm{Q}^{*}$ & ns \\
\hline
\end{tabular}

Em que: ns = não significativo; $*=$ significativo a $5 \% ; * *$ significativo a $1 \% ; \mathrm{L}=$ linear; $\mathrm{Q}=$ quadrática. 
A exigência nutricional da espécie obedeceu à seguinte ordem decrescente: $\mathrm{N}>\mathrm{Ca}>\mathrm{K}>\mathrm{Mg}$ $>\mathrm{P}>\mathrm{S}>\mathrm{Fe}>\mathrm{Mn}>\mathrm{B}>\mathrm{Zn}>\mathrm{Cu}$. Com base na maior exigência nutricional da espécie, verificouse que o $\mathrm{Ca}$ foi o segundo nutriente mais exigido, evidenciando que, para o desenvolvimento inicial de teca, a disponibilidade de cálcio em solo deve ser alta, por se tratar do nutriente que mais se acumulou nas plantas. Dessa forma, tem-se a necessidade de realizar a calagem visando suprir de modo adequado esse nutriente à cultura.

$\mathrm{O}$ maior acúmulo de $\mathrm{Ca}$ foi obtido com saturação próxima a que favoreceu a maior produção de biomassa seca de plantas de teca (saturação por bases de $60 \%$ ), demonstrando que nestas condições de saturação houve maior eficiência na utilização deste nutriente.

Os índices relativos de clorofila e a taxa de transpiração foram influenciados significativamente pelos níveis de saturação por bases (Tabela 4). Estes resultados demonstraram a importância da maior disponibilidade de nutrientes nos efeitos fisiológicos das plantas.

Para o IRC, a saturação por bases estimada de $65 \%$, segundo a equação ajustada $(\mathrm{y}=29,377$ $\left.+0,5103 x-0,0039 x^{2}\right)$ proporcionou o máximo índice. A crescente absorção de $\mathrm{Mg}$, resultante dos níveis crescentes de $\mathrm{V} \%$, pode ser a explicação para o resultado positivo do IRC. O IRC apresentou relações tanto com a concentração como com o acúmulo de $\mathrm{Mg}$ nas folhas de teca (Figura 4). O papel mais conhecido do magnésio na vida da planta refere-se à sua presença na clorofila e, segundo Malavolta et al. (1997), cerca de $10 \%$ do Mg total da planta está presente nesta molécula.

Para a taxa de transpiração, também houve efeito quadrático $\left(\mathrm{y}=6,7323+0,0365 \mathrm{x}-0,0008 \mathrm{x}^{2}\right)$, onde a saturação estimada de $23 \%$ proporcionou a máxima taxa de transpiração. A partir deste valor passou a ser decrescente em relação aos níveis crescentes de V\%. Por fazer parte da abertura e fechamento dos estômatos, o $\mathrm{K}$ na planta está diretamente ligado à taxa de transpiração, que pode ser comprovada pela relação entre a TT e o acúmulo de $\mathrm{K}$ nas folhas de teca (Figura 5). Essa relação foi negativa, ou seja, com o acúmulo de $\mathrm{K}$ houve uma redução da taxa de transpiração.

\section{CONCLUSÕES}

A correção do solo favorece todas as características de crescimento inicial (altura, diâmetro e biomassa) avaliadas nas plantas de teca.

A calagem afeta positivamente a absorção de todos os macro e micronutrientes com exceção do acúmulo de zinco. O acúmulo dos nutrientes na parte aérea da teca obedece a seguinte ordem decrescente: $\mathrm{N}>\mathrm{Ca}>\mathrm{K}>\mathrm{Mg}>\mathrm{P}>\mathrm{S}>\mathrm{Fe}>\mathrm{Mn}$ $>\mathrm{B}>\mathrm{Zn}>\mathrm{Cu}$.

$\mathrm{O}$ maior desenvolvimento das plantas e acúmulo de nutrientes fica na faixa de 60 a $80 \%$ de saturação por bases (70 \% em média). Como ação contrária, o menor desenvolvimento das plantas é resultante do tratamento sem adição da calagem na elevação da saturação por bases.

As doses crescentes de calcário aumentam o acúmulo de $\mathrm{K}$ pelas plantas de teca, reduzindo a taxa de transpiração.

TABELA 4: Índice relativo de clorofila (IRC), taxa fotossintética (TF) e taxa de transpiração (TT) coletados em 240 dap, em função de níveis crescentes de saturação por bases (V\%). BotucatuSP (julho/2009).

TABLE 4: Relative chlorophyll content (IRC), photosynthetic rate (TF) and transpiration rate (TT) collected 240 dap, due to increasing levels of base saturation (V\%). Botucatu-SP (July/2009).

\begin{tabular}{|c|c|c|c|}
\hline Níveis de saturação por bases & IRC & $\mathrm{TF}$ & TT \\
\hline ----------------\%---------------- & & $\mu \mathrm{mol} \cdot \mathrm{CO}_{2} \cdot \mathrm{m}^{-2} \cdot \mathrm{s}^{-1}$ & $\mathrm{~mol} \cdot \mathrm{H}_{2} \mathrm{O} \cdot \mathrm{m}^{-2} \cdot \mathrm{s}^{-}$ \\
\hline 0 & 27,3 & 20,1 & 6,94 \\
\hline 20 & 42,8 & 19,0 & 6,60 \\
\hline 40 & 41,6 & 23,4 & 7,34 \\
\hline 60 & 43,6 & 20,9 & 6,12 \\
\hline 80 & 46,5 & 20,0 & 4,57 \\
\hline Regressão & $\mathrm{L}^{* *}, \mathrm{Q}^{* *}$ & ns & $L^{*}, Q^{*}$ \\
\hline
\end{tabular}

Em que: ns = não significativo; $*=$ significativo a $5 \%$; * = significativo a $1 \%$; $=$ linear; $\mathrm{Q}=$ quadrática. 

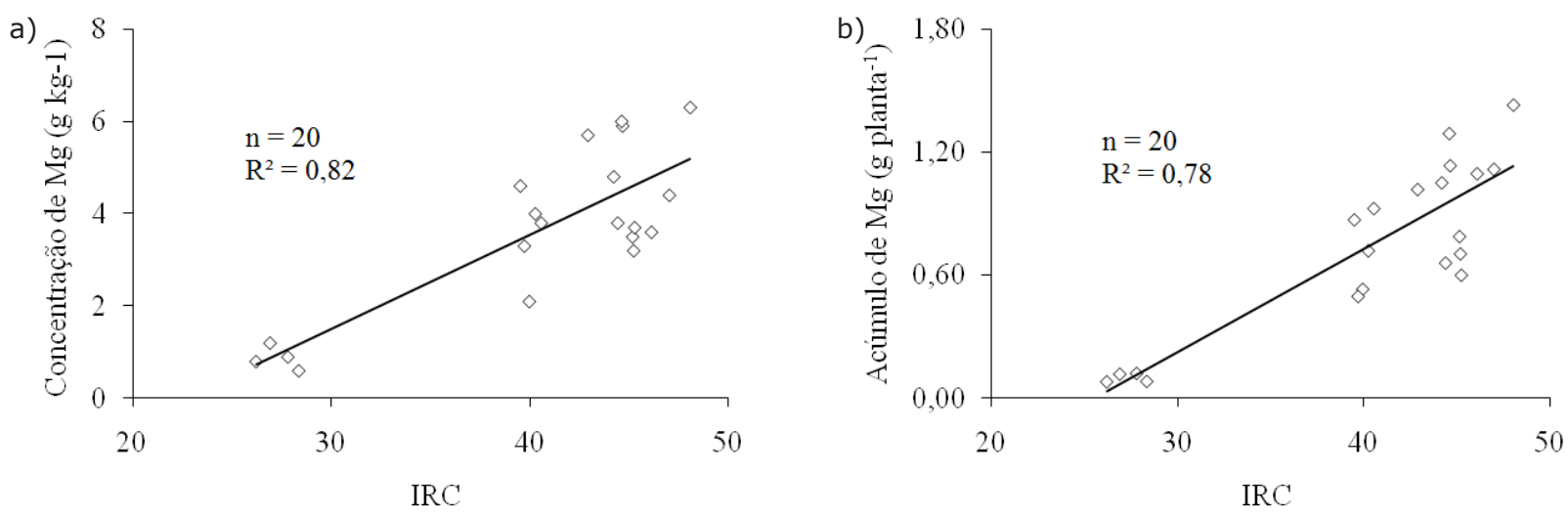

FIGURA 4: Relação entre IRC e concentração foliar de Mg (a), e acúmulo de Mg nas folhas de teca (b).

FIGURE 4: Relationship between CRI and leaf concentration of $\mathrm{Mg}$ (a) and accumulation of $\mathrm{Mg}$ in the leaves of teak (b).

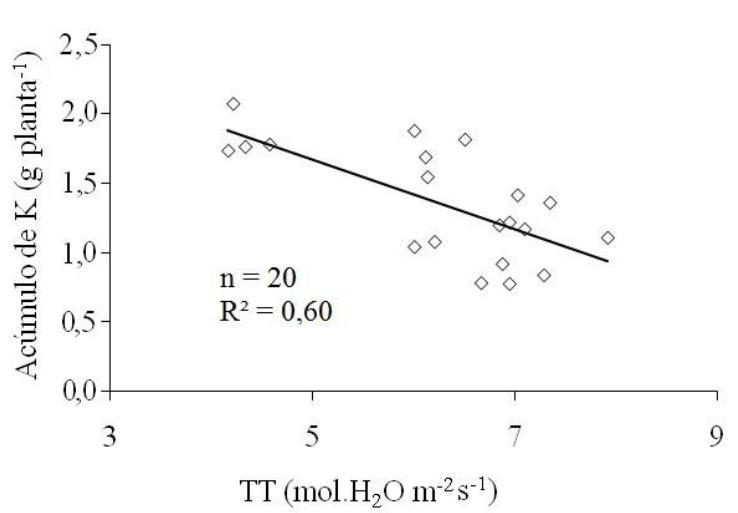

FIGURA 5: Relação entre taxa de transpiração (TT) e acúmulo de $\mathrm{K}$ nas folhas de teca.

FIGURE 5: Relationship between transpiration rate (TT) and accumulation of $\mathrm{K}$ in leaves of teak.

\section{REFERÊNCIAS BIBLIOGRÁFICAS}

BARROS,J.G.Adubação ecalagemparaformação de mudas de mogno (Swietenia macrophylla King). 2001. 63 f. Dissertação (Mestrado em Agronomia) - Universidade Federal do Amazonas, Manaus, 2001.

BARROSO, D. G. et al. Diagnóstico de deficiência de macronutrientes em mudas de Teca. Revista Árvore, Viçosa, v. 29, n. 5, p. 671 - 679, 2005.

BEHLING, M. Nutrição, partição de biomassa e crescimento de povoamentos de teca em Tangará da Serra-MT. 2009. 156 f. Tese (Doutorado em Solos e Nutrição de Plantas) - Universidade Federal de Viçosa, Viçosa, 2009.

CAMPOLIN, W. R. Características físicas e químicas, estoque de matéria orgânica (total e nas frações granulométricas) do solo sob cerrado e teca. 2006. 49 f. Dissertação (Mestrado em Agricultura Tropical) - Universidade Federal de Mato Grosso, Cuiabá2006.

CRUZ, C. A. F. et al. Efeito de diferentes níveis de saturação por bases no desenvolvimento e qualidade de mudas de ipê-roxo (Tabebuia impetiginosa (Mart.) Standley). Scientia Florestalis, Piracicaba, v. 21, n. 66, p. 100 - 107, 2004.

EMBRAPA. Centro Nacional de Pesquisa de Solos - CNPS. Sistema brasileiro de classificação de solos. 2. ed. Rio de Janeiro: Embrapa-CNPS, 2006. $306 \mathrm{p}$.

FIGUEIREDO, E. O. et al. Teca (Tectona grandis L.f.): principais perguntas do futuro empreendedor florestal. Rio Branco: Embrapa, 2005. 22 p. (Documentos, 97).

FURTINI NETO, A. E. et al. Acidez do solo, crescimento e nutrição mineral de algumas espécies arbóreas, na fase de muda. Cerne, Lavras, v. 5, n. 2, p. 1-12, 1999.

FURTUNATO, R. P. pH do solo e toxidez de alumínio no crescimento e nutrição mineral de mudas de grápia (Apuleia leiocarpa). 2001. 59 f. Dissertação (Mestrado em Engenharia Florestal) Universidade Federal de Santa Maria, Santa Maria, 2001.

MALAVOLTA, E. et al. Avaliação do estado nutricional das plantas: princípios e aplicações. 2. ed. Piracicaba: Potafos, 1997. 319 p.

MATRICARDI, W. A. T. Efeitos dos fatores do solo sobre o desenvolvimento da Teca (Tectona grandis L.f.) cultivada na grande Cáceres - Mato Grosso. 1989. 135 f. Dissertação (Mestrado em 
Ciências Florestais) - Escola Superior de Agricultura "Luiz de Queiroz", Piracicaba, 1989.

OMBINA, C. A. Soil characterization for teak (Tectona grandis L.f.) plantations in Nzara district of South Sudan. 2008. 122 f. Dissertação (Master of Forest Science) - Université des Sciences et Techniques de Masuku, GABON, 2008.

PINTO, F. P. da S. et al. Calagem e adubação na formação de mudas de mogno. In.: JORNADA DE INICIAÇÃO CIENTIFICA DA UNIVERSIDADE DO AMAZONAS, 9., 2000, Manaus. Resumos... , 2000, p. 5.

RAIJ, B. Van. Fertilidade do solo e adubação. São Paulo: Ceres/Potafos, 1991. 343 p.

RIOS, P. I. M. Estudo da viabilidade econômicofinanceira do reflorestamento com teca na região de Pau D'arco. 2007. 43 f. Trabalho de Conclusão de Curso (Agronomia) - Faculdades Integradas Departamento de Agronomia, Planaltina, 2007.

SILVA, A. R. M. da; et al. Doses crescentes de corretivos na formação de mudas de mogno (Swietenia macrophylla King). Acta Amazônica, Manaus, v. 3, n. 2, p. 195-200, 2007a.

SILVA, A. R. M. et al. Efeitos de doses crescentes de calcário na produção de mudas de sumaúma (Ceiba pentandra L. Gaertn). Floresta, Curitiba, v. 38, n. 2, p. 295-302, 2008.

SILVA, C. A. et al. Interação calagem-adubação fosfatada e sua influência nos níveis críticos de $\mathrm{P}$ e crescimento do eucalipto. Scientia Forestalis, Piracicaba, n. 73, p. 63-72, 2007 b.

SILVA, D. J. et al. Avaliação do estado nutricional, crescimento de teca (Tectona grandis L.f.) e suas relações com os fatores de sítio do solo em plantios no sudoeste de Mato Grosso, a partir da análise de componentes principais. Corumbá: EMBRAPA, CPAP, 2000. 14 p.

TUCCI, C. A. F. et al. Adubação e calagem para a formação de mudas de sumaúma (Ceiba pentandra (L.) Gaertn). Ciências Agrárias e Ambientais, Manaus, v. 11, n. 2/2, 2004.

TUCCI, C. A. F. et al. Calagem e adubação para produção de mudas de mogno (Swietenia macrophylla King). Cerne, Lavras, v. 13, n. 3, p. 299-307, 2007.

VALE, F. R. et al. Crescimento radicular de espécies florestais em solo ácido. Pesquisa Agropecuária Brasileira, Brasília, v. 31, n. 9, p. 609-616, 1996. 